Article

\title{
Programmable Spectral Filter in C-Band Based on Digital Micromirror Device
}

\author{
Yunshu Gao ${ }^{1,2} \mathbb{D}$, Xiao Chen ${ }^{1, *}$, Genxiang Chen ${ }^{1, *}$, Zhongwei Tan ${ }^{2}$, Qiao Chen ${ }^{1}$, Dezheng Dai ${ }^{1}$, \\ Qian Zhang ${ }^{1}$ and Chao $\mathrm{Yu}^{3}$ \\ 1 College of Science, Minzu University of China, Beijing 100081, China; gaoyunshu@126.com (Y.G.); \\ chenqiao0117@163.com (Q.C.); daidezheng@126.com (D.D.); qianzhang1521@163.com (Q.Z.) \\ 2 School of Electronic and Information Engineering, Beijing Jiaotong University, Beijing 100044, China; \\ zhwtan@bjtu.edu.cn \\ 3 School of Electronic Engineering, Beijing University of Posts and Telecommunications, Beijing 100876, China; \\ yu_chao@bupt.edu.cn \\ * Correspondence: xchen4399@126.com (X.C.); gxchen_bjtu@163.com (G.C.)
}

Received: 11 January 2019; Accepted: 26 February 2019; Published: 27 February 2019

check for updates

\begin{abstract}
Optical filters have been adopted in many applications such as reconfigurable telecommunication switches, tunable lasers and spectral imaging. However, most of commercialized filters based on a micro-electrical-mechanical system (MEMS) only provide a minimum bandwidth of $25 \mathrm{GHz}$ in telecom so far. In this work, the programmable filter based on a digital micromirror device (DMD) experimentally demonstrated a minimum bandwidth of $12.5 \mathrm{GHz}$ in C-band that matched the grid width of the International Telecommunication Union (ITU) G.694.1 standard. It was capable of filtering multiple wavebands simultaneously and flexibly by remotely uploading binary holograms onto the DMD. The number of channels and the center wavelength could be adjusted independently, as well as the channel bandwidth and the output power. The center wavelength tuning resolution of this filter achieved $0.033 \mathrm{~nm}$ and the insertion loss was about $10 \mathrm{~dB}$ across the entire C-band. Since the DMD had a high power handling capability $\left(25 \mathrm{KW} / \mathrm{cm}^{2}\right)$ of around 200 times that of the liquid crystal on silicon (LCOS) chip, the DMD-based filters are expected to be applied in high power situations.
\end{abstract}

Keywords: programmable spectral filter; digital micromirror device; optical switch

\section{Introduction}

To maximize the use of fiber spectral capacity and improve network efficiency, the International Telecommunication Union (ITU) G.694.1 standard has replaced the earlier G.692 specification to eliminate inefficient optical guard bands. The next generation optical network is a flexible elastic grid network that can dynamically allocate an amount of spectral resources as needed in $12.5 \mathrm{GHz}$ increments for individual channels to support different symbol rates. It appears that traditional reconfigurable optical add/drop multiplexers (ROADMs) that provide optical switching of the fixed wavelength need to be upgraded to support flexible grid channels [1]. The flexible-grid ROADMs [2] must consist of programmable channel switching devices, such as wavelength selective switches (WSSs) or flexible-grid filters.

Currently there are two main competing technologies used in commercial WSSs and filters: The micro-electrical-mechanical system (MEMS) and the liquid crystal on silicon (LCoS) spatial light modulator (SLM). With the digital micromirror device (DMD) as an electrical input, optical output MEMS performs efficient and reliable spatial light modulation. The DMD developed by Texas Instruments is an array of thousands to millions of tiny highly reflective aluminum micromirrors 
which can be addressed independently. It has the advantage of low cost, high power handling and a fast frame rate, so that it has become an attractive solution for modulating spectrum resources in reconfigurable telecommunication switches [3,4], dynamic spatial and image filters $[5,6]$, tunable lasers [7-10] and equalizers for erbium-doped fiber amplifiers (EDFAs) [11]. However, DMD and MEMS based reconfigurable optical filters [12-14] up to date have the minimum spectral bandwidth of $25 \mathrm{GHz}$ in C-band (The TrueFlex Twin Multi-Cast Switch produced by Lumentum), so that they cannot adapt to the development of the new ITU-T standard grid frequencies. Although it was reported LCoS-based WSSs $[15,16]$ have achieved $12.5 \mathrm{GHz}$ or even smaller minimum bandwidth and higher tuning resolution, the LCOS processor under high power operation starts to deteriorate in function, even acquiring permanent, irreparable damage [17]. In comparison, the DMD has a high power handling capability and the only limit of the device under high power illumination is that the aluminum micromirrors must operate below $150^{\circ} \mathrm{C}$ [18]. Therefore, it is expected to greatly broaden the application of DMD based optical filters in high power situations.

In this paper, we employ a DMD combining with a high-line-density transmission grating into a 2- $f$ optical system to demonstrate the programmable spectral filter with flexible center wavelength, elastic bandwidth and high power handling. The minimum bandwidth achieved was $12.5 \mathrm{GHz}$. This optical filter can become a pre-filter to obtain target spectrum or an equalizer for EDFAs. It is important to compensate lower power handling of LCoS-based switches in an optical network.

\section{System Design}

Figure 1 illustrates the layout of the DMD-based optical filter which consists of a fiber-coupling microlens array, a polarization converter, two lenses, a transmission grating and a DMD. Two ports from the fiber-coupling microlens array with a $127 \mu \mathrm{m}$-pitch were used as an input and an output. For the high-line-density transmission grating with 1201.2 line/mm is S-polarization dependent, a polarization converter was inserted after the micro-lens to modulate the polarization state of an input beam. The DMD adopted in system consisted of $1024 \times 768$ mirrors on a pitch of $\delta=13.68 \mu \mathrm{m}$ with $\pm 12^{\circ}$ micromirror tilt by software control. It had a highly efficient steering of NIR light and an anti-reflection coated substrate which assured a front cover reflection of less than $0.5 \%$ between 1400 and $1700 \mathrm{~nm}$.

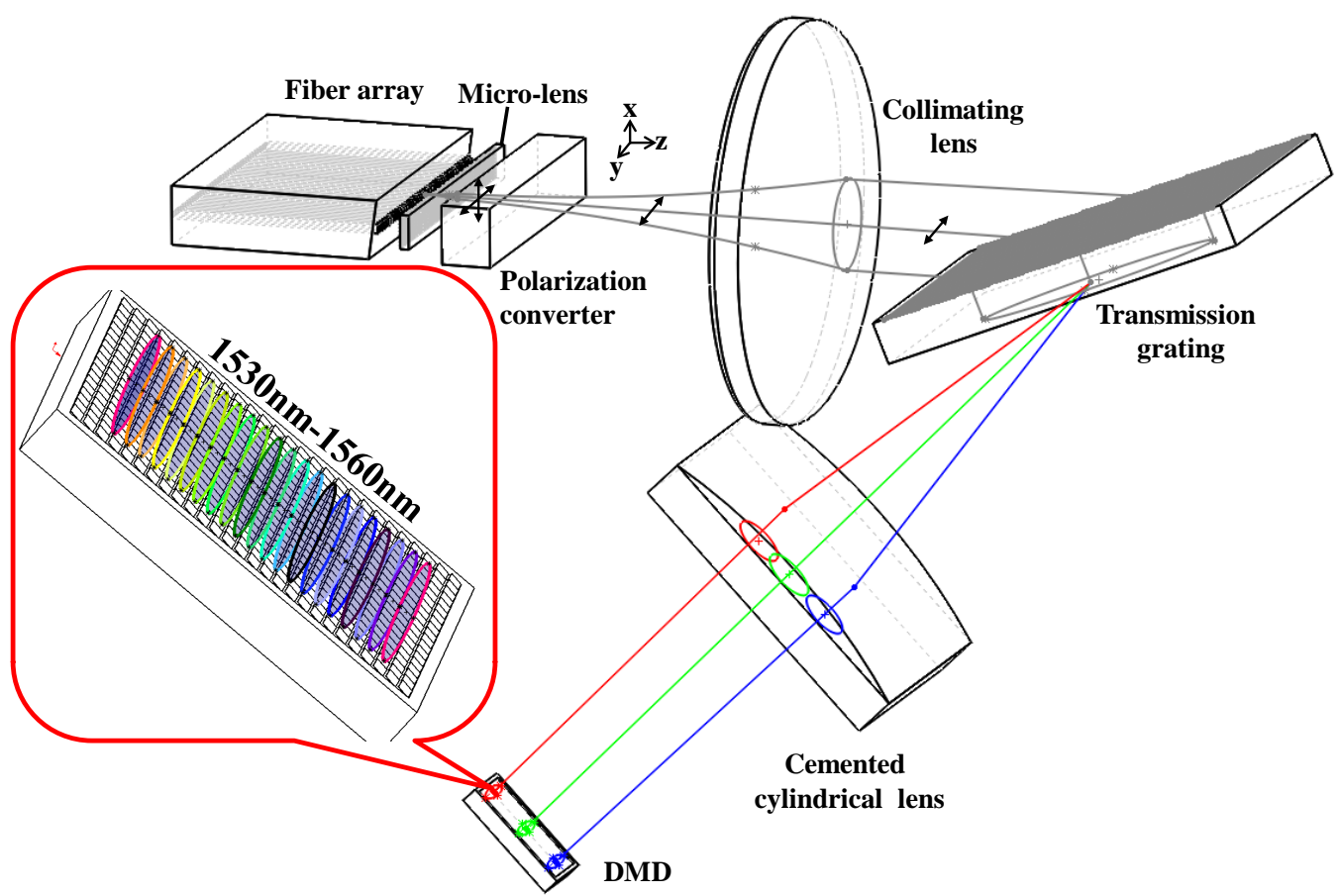

Figure 1. Diagram of programmable optical filter based on the digital micromirror device (DMD) chip. 
Figure $2 \mathrm{a}$ is the $x z$-plane view and Figure $2 \mathrm{~b}$ is the $y z$-plane view. We simplify the optical configuration by ignoring the angle of a transmission grating, so that the optical axis shown in Figure 2 is a straight line. A microlens combining with a collimating lens converted an input divergent Gaussian beam into a $6 \mathrm{~mm}$-diam parallel beam $[19,20]$. The collimated broad-band beam was angularly dispersed in the $x$-axis direction by a grating and then focused into an elliptical spot on a different area of the DMD after a cylindrical lens. The purpose of the elliptical spot was to obtain the minimum bandwidth and high diffraction efficiency [16]. The DMD was placed at the focal plane of both the cylindrical lens $\left(f_{2}=140 \mathrm{~mm}\right)$ and the collimating lens $\left(f_{1}=300 \mathrm{~mm}\right)$ to realize the function that each micromirror was controlled at the on or off states to select and steer arbitrary wavebands precisely to the output.

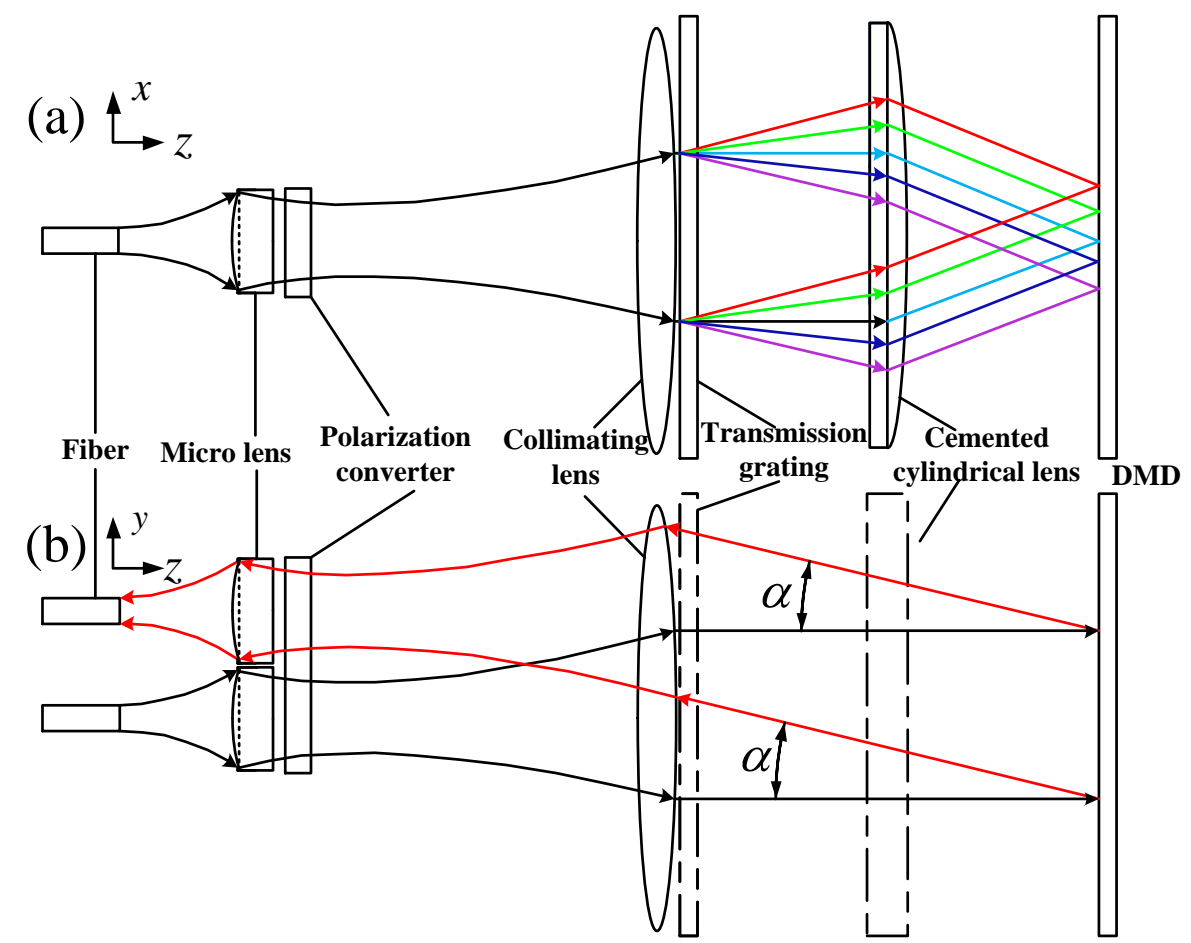

Figure 2. Layout of the filter optics: (a) The view in $x z$-plane showing light being de-multiplexed (b) The view in yz-plane showing light deflected by a DMD.

\subsection{Diffraction Efficiency of DMD}

In general, the long side of the DMD chip is aligned with the dispersion strip along the $x$-axis direction to maximize the spectrum utilization. The binary amplitude grating patterns are uploaded onto the DMD to control the corresponding micromirrors to tilt $\pm 12^{\circ}$ angle along their diagonals. The diffraction behavior of the several hundred thousand individually tilted micromirrors array as shown in Figure 3a is similar to a two-dimensional blazed grating. The diffraction distribution by the DMD and the coordinate system $\left(x_{0}, y_{0}, z_{0}\right)$ is established in Figure $3 \mathrm{~b}$. The blue line represents an input beam and the red lines are the corresponding high-order diffraction beams in space. According to the 2D diffraction model [10], the diffraction angle of each order $(p, q)$ is written as:

$$
\left\{\begin{array}{l}
\phi_{\text {out }}(p, q)=\tan ^{-1}\left(\frac{H(q)}{G(p)}\right) \\
\theta_{\text {out }}(p, q)=\sin ^{-1}\left(\frac{G(p)}{\cos \phi_{\text {out }}(p, q)}\right),
\end{array}\right.
$$

where $H(q)=\frac{q \lambda}{\delta}+\sin \theta_{\text {in }} \cos \phi_{i n}, G(p)=\frac{p \lambda}{\delta}+\sin \theta_{i n} \cos \phi_{i n} . \phi_{i n}$ is the incident angle between the input plane and $y_{0}$-axis, $\theta_{i n}$ is the angle between the incident beam and $z_{0}$-axis in output plane as shown in Figure $3 c$. $\phi_{\text {out }}$ and $\theta_{\text {out }}$ are defined in the same way. In Equation $(1),(p, q)=0, \pm 1, \pm 2, \cdots$ 
represent different diffraction orders. The diffraction distribution of an arbitrary order can be obtained when the incident angle $\phi_{i n}$ and $\theta_{\text {in }}$ are provided.
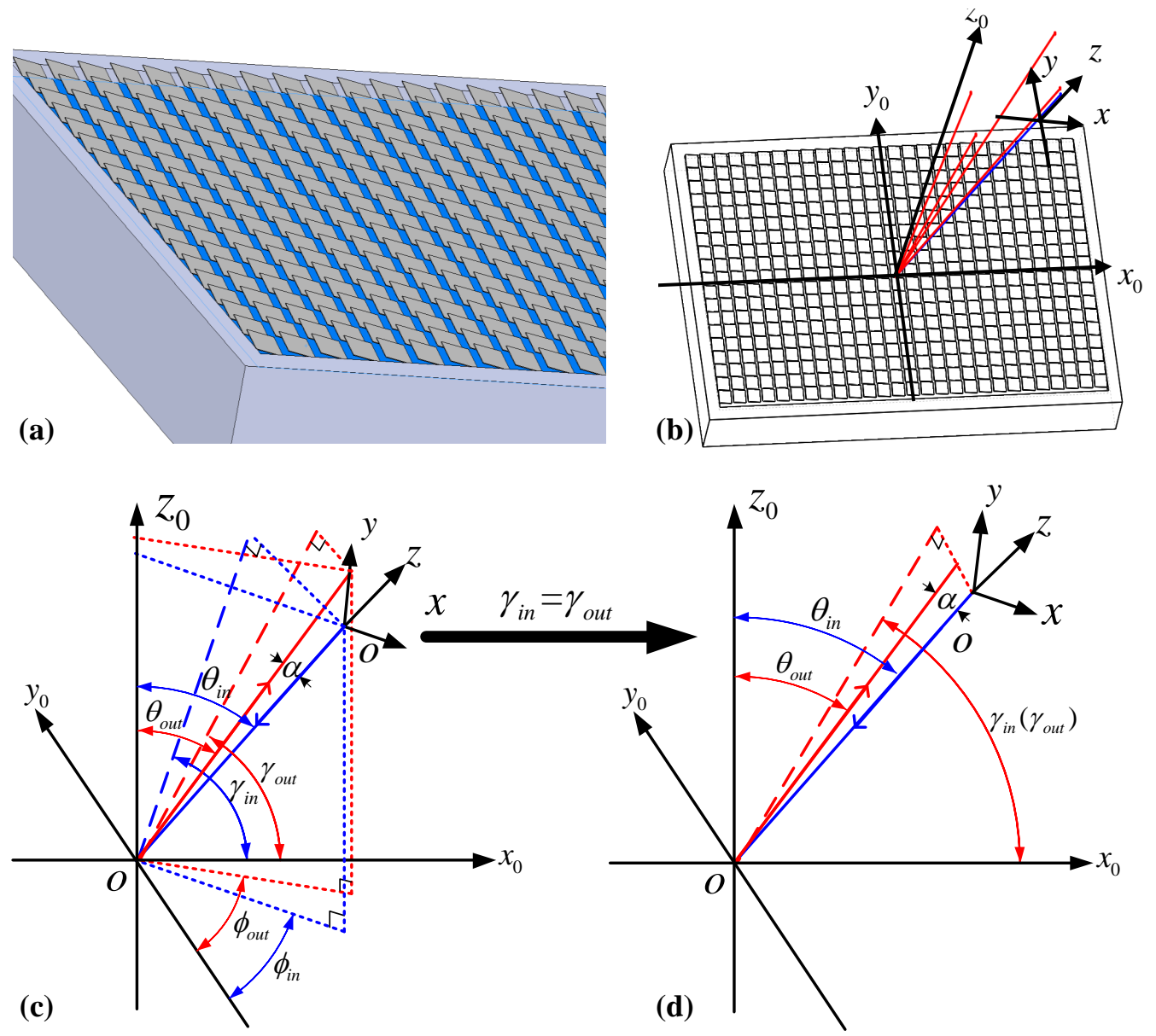

Figure 3. (a) Two-dimensional diffraction model of the DMD. (b) The incident beam and diffraction beam. (c) The coordinate system $\left(x_{0}, y_{0}, z_{0}\right)$. (d) Distribution of the input and output beam when $\gamma_{i n}=\gamma_{o u t}$.

As shown in Figure $3 c$, since the port distribution direction is perpendicular to the spectrum dispersion direction that is the $x_{0}$-axis direction, the incident angle needs to be adjusted to ensure the diffraction order with highest intensity is located in yoz-plane and routed back into an output port. The angle $\alpha$ is defined as that between input beam and diffraction beam. It is necessary to ensure $\gamma_{\text {in }}=\gamma_{\text {out }}$ in Figure $3 \mathrm{~d}$, with $\gamma_{\text {in }}$ and $\gamma_{\text {out }}$ being two angles between the projection of diffraction beam in $x_{0} o z_{0}$-plane and $x_{0}$-axis respectively. So the incident angles $\left(\theta_{i n}, \phi_{i n}\right)$ satisfy the following condition:

$$
\frac{\cos \theta_{\text {in }}}{\sin \theta_{\text {in }} \sin \phi_{\text {in }}}=\frac{\cos \theta_{\text {out }}}{\sin \theta_{\text {out }} \sin \phi_{\text {out }}} .
$$

Based on the 2D diffraction model above, when a $1550 \mathrm{~nm}$ beam radiates on the $+12^{\circ}$ tilt DMD, it is noticed that the $(-3,-3)$-order diffraction beam always has a higher efficiency than the others. So multiple optimal incident angles according to Equations (1) and (2) are calculated and shown in Figure $4 \mathrm{a}$. The angle $\alpha$ and normalized diffraction intensity of the $(-3,-3)$-order diffraction beam as a function of incident angles are presented in Figure $4 b, c$, respectively. Although the maximum normalized diffraction intensity can achieve 55\%, the larger angle $\alpha=2.5^{\circ}$ worsens the optical aberration and insertion loss. So $\alpha$ is controlled at about $1^{\circ}$ when $\theta_{\text {in }}=13.91^{\circ}, \phi_{\text {in }}=42^{\circ}$. 

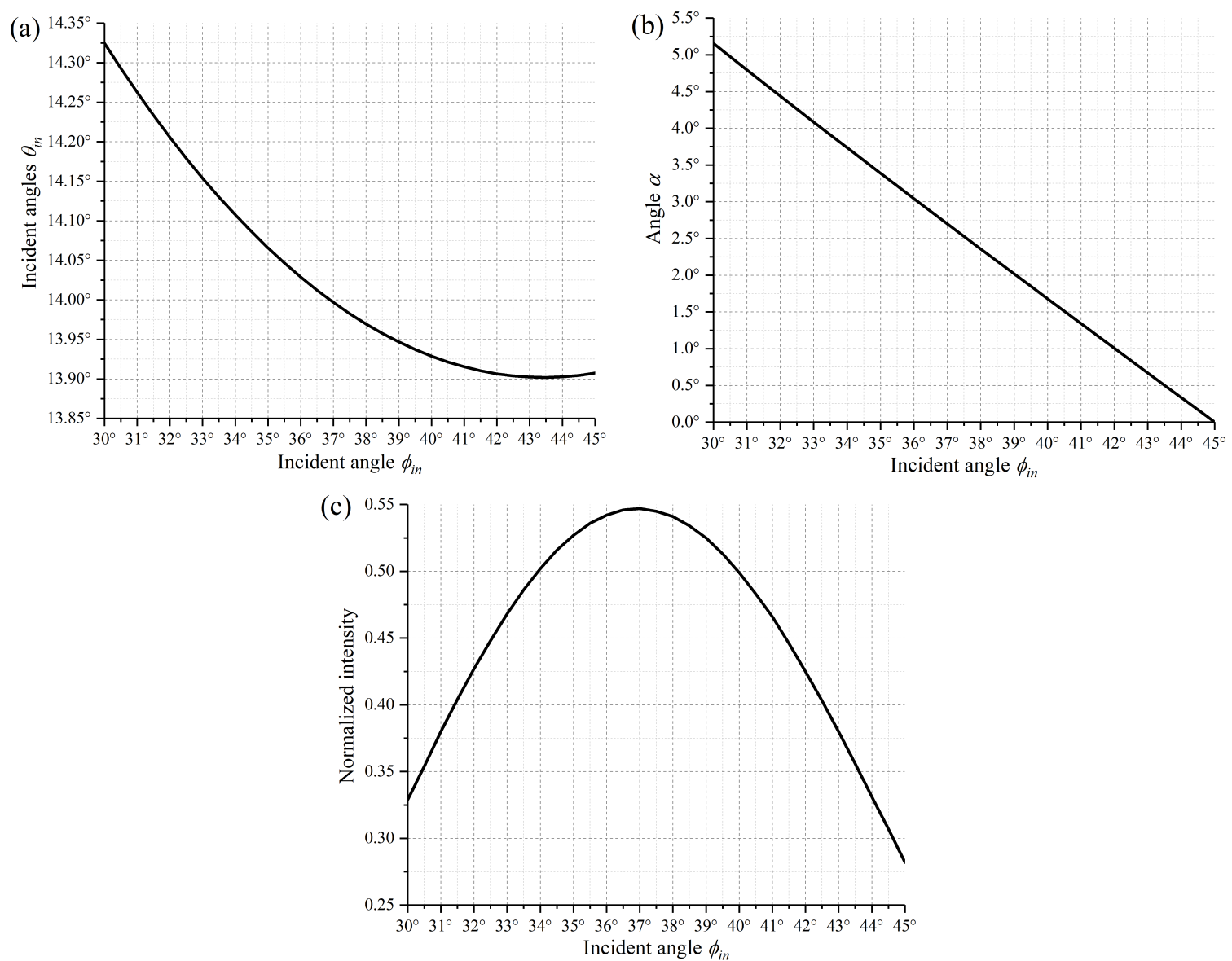

Figure 4. (a) Optimal incident angles $\left(\theta_{i n} / \phi_{\text {in }}\right)$ for the optical system. (b) Dependence of the angle $\alpha$ on the incident angles $\theta_{\text {in }}$. (c) Dependence of the normalized intensity of output beam on incident angles $\theta_{\text {in }}$.

Table 1 gives the diffraction principal maximum beam when a $1550 \mathrm{~nm}$ beam radiating on the DMD at $\theta_{\text {in }}=13.91^{\circ}, \phi_{\text {in }}=42^{\circ}$, including the corresponding diffraction angle $\left(\theta_{\text {out }} / \phi_{\text {out }}\right)$ and relative intensity $I(\theta, \phi)$ of each $(p, q)$-order. The brightest order of diffracted light is $I(-3,-3)=0.425$, so that it is selected to couple into the output port while the other peaks are dramatically dropped out. The insertion loss by the DMD diffraction is around $3.7 \mathrm{~dB}$.

Table 1. Irradiance maxima of light at $1550 \mathrm{~nm}$ radiating on the DMD over a large solid angle $\theta_{\text {out }} / \phi_{\text {out }}$, (I) of each diffraction order.

\begin{tabular}{ccccc}
\hline$p / q$ & $\mathbf{- 4}$ & $\mathbf{- 3}$ & $\mathbf{- 2}$ & $\mathbf{- 1}$ \\
\hline \multirow{4}{*}{4} & $22.8 / 45$ & $18.6 / 30.4$ & $16.1 / 9.9$ & - \\
& $(0.002)$ & $(0.035)$ & $(0.005)$ & - \\
-3 & $18.6 / 59.6$ & $\mathbf{1 3 . 2 / 4 5}$ & $9.7 / 16.5$ & $10.0 /-22.1$ \\
& $(0.019)$ & $\mathbf{( 0 . 4 2 5 )}$ & $(0.05)$ & $(0.009)$ \\
-2 & $16.1 / 80.1$ & $9.7 / 73.5$ & $3.872 / 45$ & $4.7 /-54$ \\
& $(0.011)$ & $(0.213)$ & $(0.018)$ & $(0.004)$ \\
-1 & $-16.4 /-76.6$ & $-10.0 /-67.9$ & $-4.7 /-36.1$ & - \\
& $(0.001)$ & $(0.03)$ & $(0.003)$ & - \\
\hline
\end{tabular}

\subsection{Power Handling of Optical filter}

The power handling is one of the important specifications of optical filters. When the continuous wave (CW) laser illuminates a DMD, excessive energy absorption by on-surface aluminium-mirrors generally leads to the abnormal operation or even irreversible damage of the device. Therefore, it is 
necessary to keep the operating temperature below a critical point of $150{ }^{\circ} \mathrm{C}$, and the average intensity cannot exceed $25 \mathrm{KW} / \mathrm{cm}^{2}$ in the visible band [18]. In general, the damage threshold depends on the illumination wavelength and intensity profile. For example, a damage threshold for $1550 \mathrm{~nm}$ is twice of $645 \mathrm{~nm}$. As shown in Figure 5, a Gaussian beam has a maximum power density twice of the uniform beam when both beams have the same spot size and power. It is reported the damage threshold of the DMD for $1550 \mathrm{~nm}$ Gaussian beam is estimated to be about $25 \mathrm{KW} / \mathrm{cm}^{2}$ for CW-laser. Faustov [21] demonstrated that the measured threshold is up to $22 \mathrm{~mW}$ corresponding to $12 \mathrm{KW} / \mathrm{cm}^{2}$ when a He-Ne laser at $633 \mathrm{~nm}$ is focused onto a $13.7 \mu \mathrm{m} \times 13.7 \mu \mathrm{m}$-size micromirror. Furthermore, when an input laser at $1064 \mathrm{~nm}$ is below $30 \mathrm{~mW}\left(21 \mathrm{KW} / \mathrm{cm}^{2}\right)$, micromirrors do not exhibit any visible damage. Schwarz et al. [22] also experimentally showed the damage threshold of a $19.3 \mathrm{KW} / \mathrm{cm}^{2} \mathrm{by}$ 532 nm CW-laser.

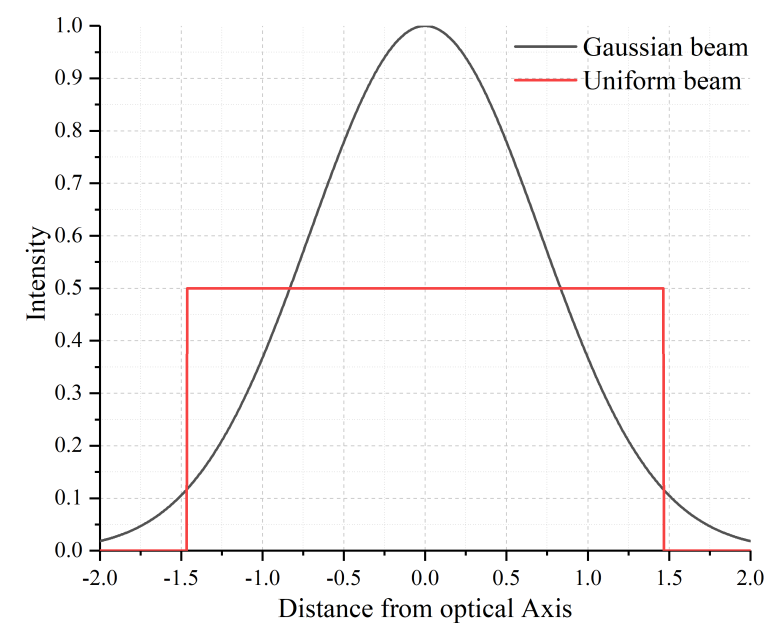

Figure 5. The beam intensity distribution of the Gaussian beam and uniform beam.

The compressed light spot on the DMD means not only a narrower bandwidth for the filter, but also a higher energy density. The spot size on the DMD for this filter is measured to be $60 \mu \mathrm{m} \times 9 \mathrm{~mm}$, so the max input power is about $135 \mathrm{~W}(50 \mathrm{dBm})$ corresponding to $25 \mathrm{KW} / \mathrm{cm}^{2}$. The power handling of commercialized WSSs (Waveshaper 16,000 A produced by Finisar Corporation) and LCoS based filters is $27 \mathrm{dBm}$ input power in maximum. Therefore, DMD-based filters are an irreplaceable solution in high power situation.

\section{Experimental Results and Discussion}

Figure 6 is the arrangement of the optical filter in experiment. The amplified spontaneous emission (ASE) light source in 1530-1560 nm was injected into the system as input signals. An optical spectrum measurement analyzer AQ6370C-YOKOGAWA was applied to measure the insertion loss, $3 \mathrm{~dB}$-bandwidth and tuning resolution of the central wavelength. Figure 7 shows that the total loss was around $10 \mathrm{~dB}$ across the entire C-band with the ripples of $0.5 \mathrm{~dB}$ caused by the gap between micromirrors. The insertion loss mainly included $1.5 \mathrm{~dB}$ from the fiber-coupling microlens array, $0.5 \mathrm{~dB}$ from the polarization converter, $1.0 \mathrm{~dB}$ from the transmission grating and $4 \mathrm{~dB}$ from the DMD. In addition, when incident angle of the input beam at $\theta_{\text {in }}=13.91^{\circ}, \phi_{\text {in }}=42^{\circ}$, a mismatch between the focal plane of the cylindrical lens and the DMD caused $3 \mathrm{~dB}$ extra insertion loss. Although this $3 \mathrm{~dB}$ loss caused by oblique incident beam could be avoided by replacing the DMD with a $10.8 \mu$ m-pitch micromirror that had a $98 \%$ diffraction efficiency to vertical incident beam [10], it was not applied in the NIR-band without an anti-reflection coating, which would introduce more loss. The measured intrinsic polarization dependent loss (PDL) within the $12.5 \mathrm{GHz}$-bandwidth was less than $1 \mathrm{~dB}$ for the optical system. As the signal beam was diffracted by the transmission grating, a combination of conical diffraction and optical aberrations lead to the fluctuation of insertion loss of about $1 \mathrm{~dB}$ [16]. 


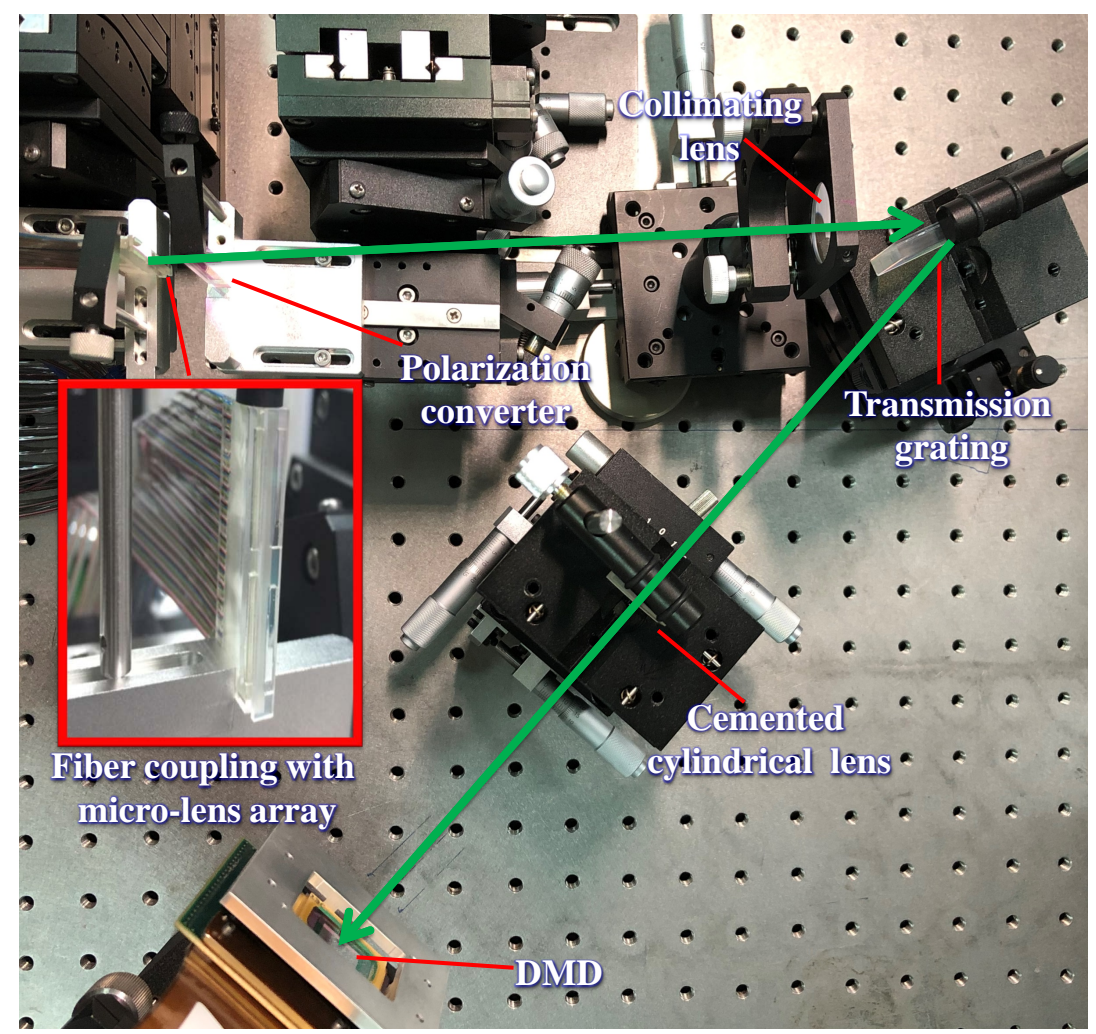

Figure 6. Arrangement of tunable optical filter.

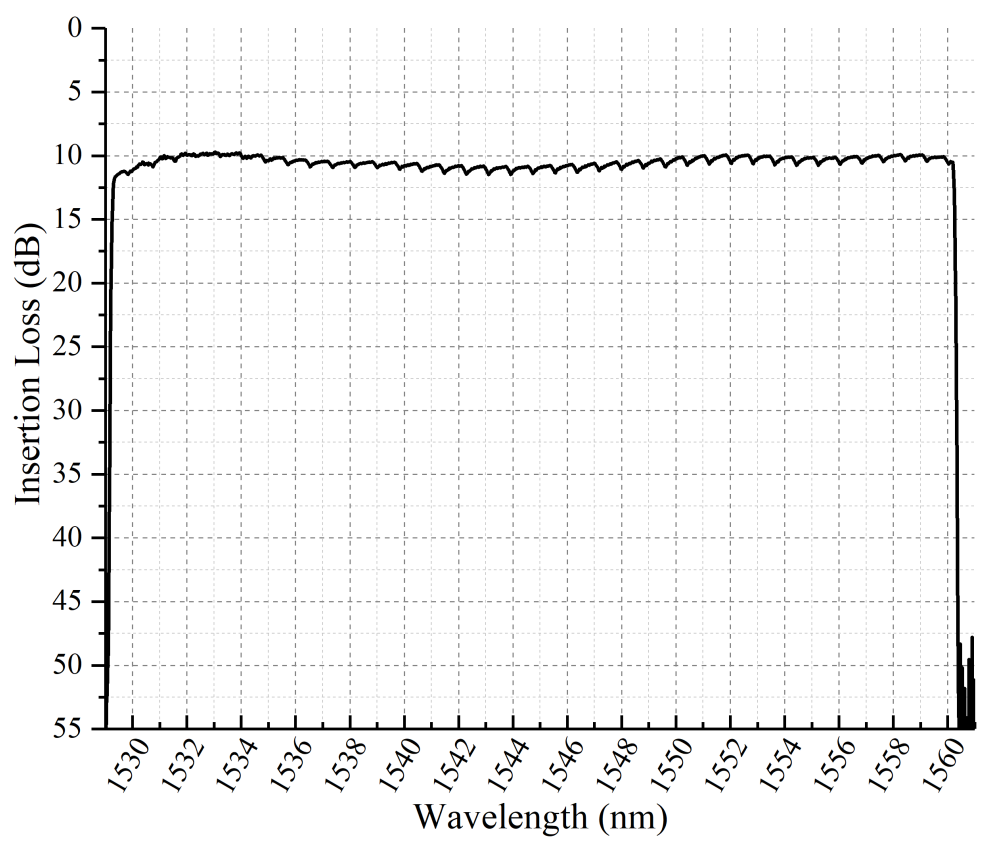

Figure 7. Total insertion loss as a function of C-band wavelength in filter system.

Figure 8 a shows that the center wavelength could be tuned flexibly in step of $0.033 \mathrm{~nm}$ with the 3 dB-bandwidth of $12.5 \mathrm{GHz}$. In Figure $8 \mathrm{~b}$, arbitrary wavebands could be filtered and configured at a minimum resolution of $0.033 \mathrm{~nm}$. The $3 \mathrm{~dB}$-passband could be adjusted flexibly from $12.5 \mathrm{GHz}$ to $50 \mathrm{GHz}$ by a step of $12.5 \mathrm{GHz}$ in Figure 8c. The minimum filter bandwidth could achieve $12.5 \mathrm{GHz}$, however it was noticed that the top of wavelength profile was not flat enough. The measured passband 
at nine ITU-T G.694.1 standard grid frequencies with $25 \mathrm{GHz}$ channel separation had about $-15 \pm 1 \mathrm{~dB}$ channel crosstalk in Table 2 and Figure 8d. Since the power falling edge of this filter was about $12 \mathrm{GHz}$ spectral width in $20 \mathrm{~dB}$, very narrowing spectral guard bands with small crosstalk could be set for $50 \mathrm{GHz}$ and $100 \mathrm{GHz}$ spaced ITU-T channels in the C-band.

Table 2. Channel crosstalk and offset level at nine grid frequencies ( $25 \mathrm{GHz}$ channel separation).

\begin{tabular}{cccccccccc}
\hline $\begin{array}{c}\text { Center } \\
\text { Wavelength } \\
(\mathbf{n m})\end{array}$ & $\mathbf{1 5 5 0 . 0 9}$ & $\mathbf{1 5 5 0 . 3 0}$ & $\mathbf{1 5 5 0 . 5 0}$ & $\mathbf{1 5 5 0 . 7 1}$ & $\mathbf{1 5 5 0 . 9 1}$ & $\mathbf{1 5 5 1 . 1 1}$ & $\mathbf{1 5 5 1 . 3 2}$ & $\mathbf{1 5 5 1 . 5 3}$ & $\mathbf{1 5 5 1 . 7 4}$ \\
\hline $\begin{array}{c}\text { Offset } \\
\text { Level (dB) } \\
\begin{array}{c}\text { Channel } \\
\text { Crosstalk (dB) }\end{array}\end{array}$ & -0.557 & -0.25 & -0.067 & -0.029 & -0.502 & -0.267 & -0.124 & 0 & -0.419 \\
\hline
\end{tabular}
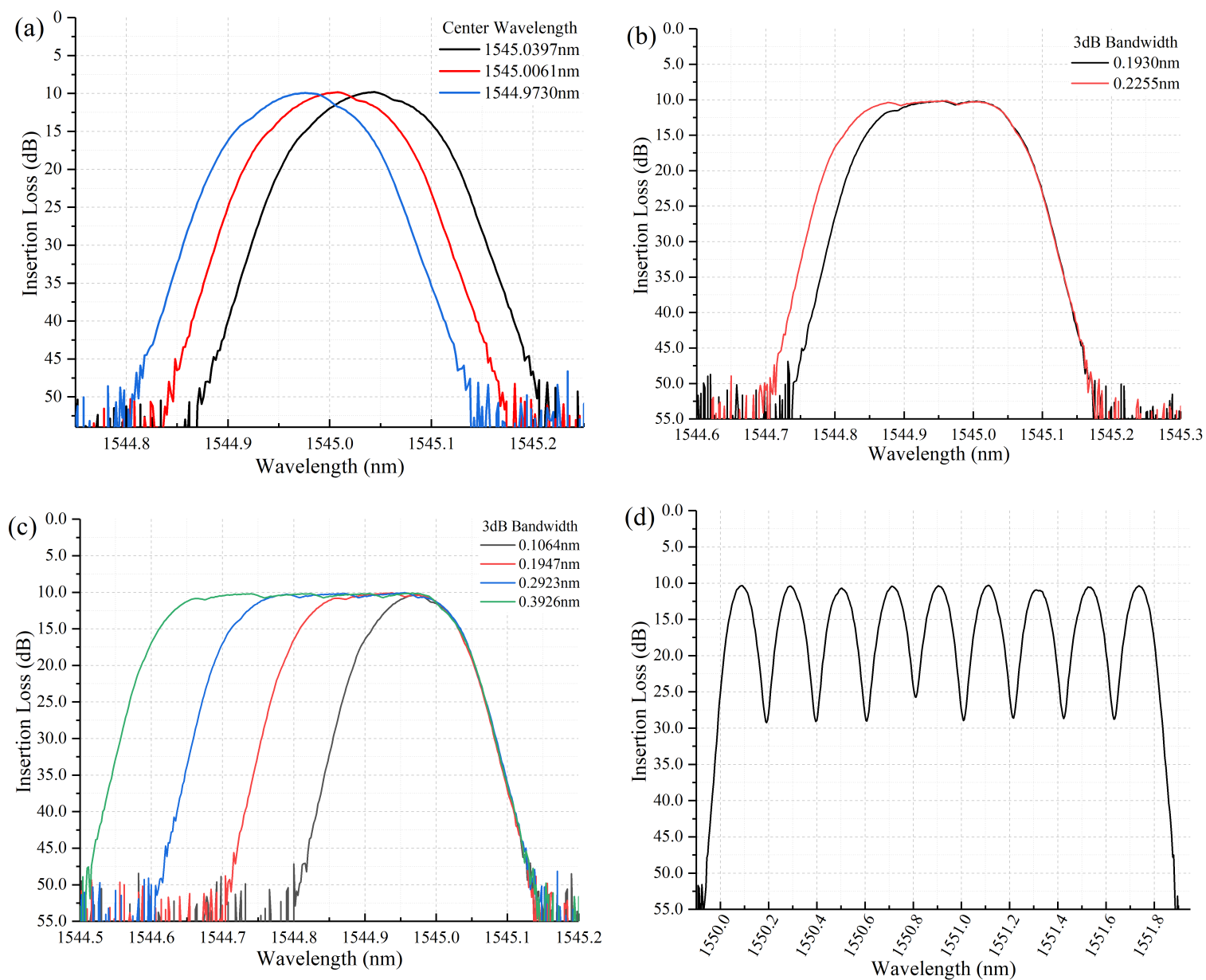

Figure 8. (a) The minimum tuning resolution of center wavelength in the filter. (b) The minimum tuning step of spectral bandwidth. (c) $3 \mathrm{~dB}$-bandwidth from $12.5 \mathrm{GHz}$ to $50 \mathrm{GHz}$ with a step of $12.5 \mathrm{GHz}$. (d) Measured passband at 9 G.694.1 standard grid frequencies with $25 \mathrm{GHz}$ channel separation.

In Figure 9a, the optical filter also provided a function of optical power attenuation. It was realized by controlling the corresponding micromirrors number in different locations to modulate the output luminous flux. The optical power attenuation could be adjusted from $0 \mathrm{~dB}$ to $40 \mathrm{~dB}$ flexibly with a resolution of $0.5 \mathrm{~dB}$. Figure $9 \mathrm{~b}$ shows the micromirrors information used to control the optical attenuation. This filter had $50 \mathrm{dBm}$ maximum input power, and was an excellent equalizer for high power erbium-doped fiber amplifiers. 


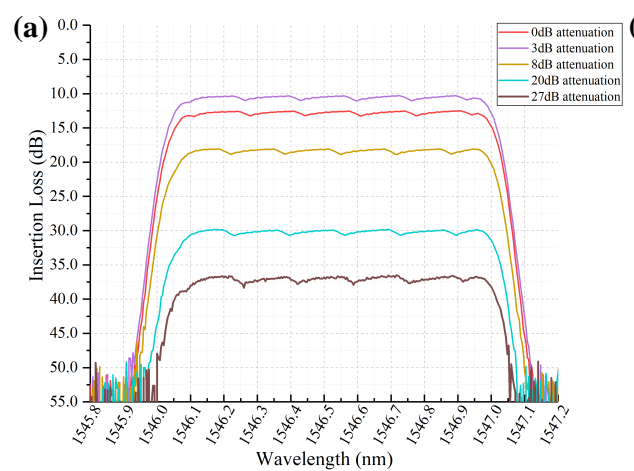

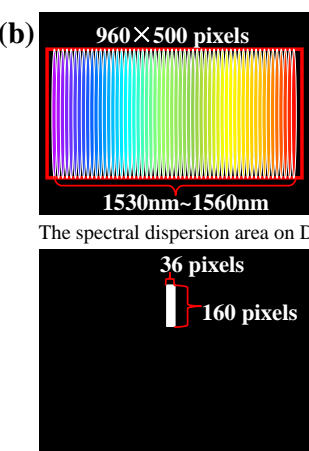

8dB attenuation
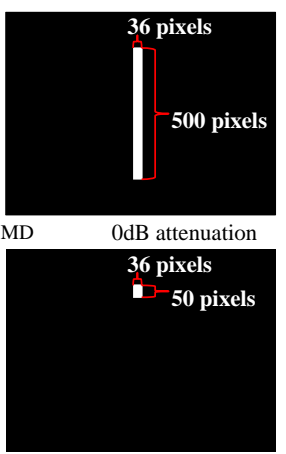

$20 \mathrm{~dB}$ attenuation

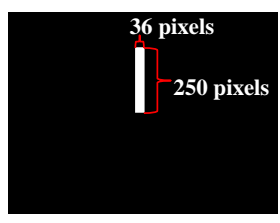

$3 \mathrm{~dB}$ attenuation

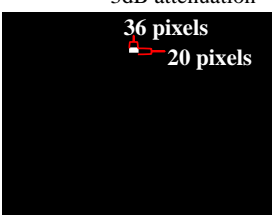

$27 \mathrm{~dB}$ attenuation

Figure 9. Optical power attenuation of the optical filter and the corresponding binary image.

\section{Conclusions}

We propose and demonstrate a tunable optical filter with max $50 \mathrm{dBm}$ input power, flexible central wavelength and bandwidth by employing a DMD processor into the system. The total insertion loss of this filter was about $10 \mathrm{~dB}$ across the entire C-band. The center wavelength and bandwidth of multi-channel could be tuned in the step of $0.033 \mathrm{~nm}$ independently. Although the minimum bandwidth could achieve $12.5 \mathrm{GHz}$, the performance of the channel crosstalk for $12.5 \mathrm{GHz}$ and $25 \mathrm{GHz}$ ITU grid especially still needs further improvement, as the spectral does not have an ideal flat-topped profile. In future work, we plan to optimize the minimum bandwidth by employing a specially designed cylindrical lens system to eliminate the spherical aberration and chromatic aberration, and further decrease the spot size in the $x$-axis on the DMD.

Author Contributions: Conceptualization, G.C.; formal analysis, Y.G., Q.C., Z.T. and D.D.; investigation, C.Y. and Q.Z.; methodology, X.C.; project administration, X.C.; writing-original draft preparation, Y.G.; writing-review and editing, X.C.

Acknowledgments: This work was supported by the National Natural Science Foundation of China Grant number 61627814, 61275052 .

Conflicts of Interest: The authors declare no conflict of interest. The funders had no role in the design of the study; in the collection, analyses, or interpretation of data; in the writing of the manuscript, or in the decision to publish the results.

\section{References}

1. Anuj Malik, G.H. The Evolution of Next-Gen Optical Networks: Terabit Super-Channels and Flexible Grid ROADM Architectures. In Proceedings of the SCTE Cable-Tec Expo 2014, Denver, CO, USA, 22-25 September 2014.

2. Woodward, S.L.; Feuer, M.D. Benefits and requirements of flexible-grid ROADMs and networks. IEEE/OSA J. Opt. Commun. Netw. 2013, 5, A19-A27. [CrossRef]

3. Blanche, P.A.; Carothers, D.; Wissinger, J.; Peyghambarian, N. DMD as a diffractive reconfigurable optical switch for telecommunication. In Proceedings of the Emerging Digital Micromirror Device Based Systems and Applications V, San Francisco, CA, USA, 5-6 February 2013.

4. Knapczyk, M.T.; de Peralta, L.G.; Bernussi, A.A.; Temkin, H. Reconfigurable add-drop optical filter based on arrays of digital micromirrors. J. Lightw. Technol. 2008, 26, 237-242. [CrossRef]

5. Jin, D.; Zhou, R.; Yaqoob, Z.; So, P.T. Dynamic spatial filtering using a digital micromirror device for high-speed optical diffraction tomography. Opt. Express 2018, 26, 428-437. [CrossRef] [PubMed]

6. Hoover, R.; Henry, A.; Arce, G.R. DMD-based implementation of patterned optical filter arrays for compressive spectral imaging. J. Opt. Soc. Am. A Opt. Image Sci. Vis. 2015, 32, 80-89.

7. Liu, W.; Fan, J.; Xie, C.; Song, Y.; Gu, C.; Chai, L.; Wang, C.; Hu, M. Programmable controlled mode-locked fiber laser using a digital micromirror device. Opt. Lett. 2017, 42, 1923-1926. [CrossRef] [PubMed]

8. Luo, D.; Taphanel, M.; Längle, T.; Beyerer, J. Programmable light source based on an echellogram of a supercontinuum laser. Appl. Opt. 2017, 56, 2359-2367. [CrossRef] [PubMed] 
9. Wood, T.C.; Elson, D.S. A tunable supercontinuum laser using a digital micromirror device. Meas. Sci. Technol. 2012, 23, 105204. [CrossRef]

10. Chen, X.; Yan, B.B.; Song, F..; Wang, Y.Q.; Xiao, F.; Alameh, K. Diffraction of digital micromirror device gratings and its effect on properties of tunable fiber lasers. Appl. Opt. 2012, 51, 7214-7220. [CrossRef] [PubMed]

11. Gu, C.; Chang, Y.; Zhang, D.; Cheng, J.; Chen, S.C. Femtosecond laser pulse shaping at megahertz rate via a digital micromirror device. Opt. Lett. 2015, 40, 4018-4021. [CrossRef] [PubMed]

12. Khan, S.A.; Riza, N.A. Demonstration of the MEMS Digital Micromirror Device-Based Broadband Reconfigurable Optical Add-Drop Filter for Dense Wavelength-Division-Multiplexing Systems. J. Lightw. Technol. 2007, 25, 520-526. [CrossRef]

13. Knapczyk, M.; Krishnan, A.; de Peralta, L.G.; Bernussi, A.; Temkin, H. High-resolution pulse shaper based on arrays of digital micromirrors. IEEE Photonics Technol. Lett. 2005, 17, 2200-2202. [CrossRef]

14. Knapczyk, M.; Krishnan, A.; de Peralta, L.G.; Bernussi, A.; Temkin, H. Reconfigurable optical filter based on digital mirror arrays. IEEE Photonics Technol. Lett. 2005, 17, 1743-1745. [CrossRef]

15. Xie, D.; Wang, D.; Zhang, M.; Liu, Z.; You, Q.; Yang, Q.; Yu, S. LCoS-based wavelength-selective switch for future finer-grid elastic optical networks capable of all-optical wavelength conversion. IEEE Photon. J. 2017, 9, 1-12. [CrossRef]

16. Gao, Y.; Chen, G.; Chen, X.; Zhang, Q.; Chen, Q.; Zhang, C.; Tian, K.; Tan, Z.; Yu, C. High-Resolution Tunable Filter With Flexible Bandwidth and Power Attenuation Based on an LCoS Processor. IEEE Photonics J. 2018, 10, 1-8. [CrossRef]

17. Carbajo, S.; Bauchert, K. Power handling for LCoS spatial light modulators. In Proceedings of the Laser Resonators, Microresonators, and Beam Control XX, San Francisco, CA, USA, 29 January-1 February 2018.

18. Laser Power Handling for DMDs. Available online: http://www.ti.com/lit/wp/dlpa027/dlpa027.pdf (accessed on 26 February 2019).

19. Iwama, M.; Takahashi, M.; Kimura, M.; Uchida, Y.; Hasesawa, J.; Kawahara, R.; Kagi, N. LCOS-based flexible grid $1 \times 40$ wavelength selective switch using planar lightwave circuit as spot size converter. In Proceedings of the 2015 Optical Fiber Communications Conference and Exhibition (OFC), Los Angeles, CA, USA, 22-26 March 2015; pp. 1-3.

20. Suzuki, K.; Ikuma, Y.; Hashimoto, E.; Yamaguchi, K.; Itoh, M.; Takahashi, T. Ultra-high port count wavelength selective switch employing waveguide-based I/O frontend. In Proceedings of the Optical Fiber Communication Conference, Los Angeles, CA, USA, 22-26 March 2015.

21. Faustov, A.R.; Webb, M.R.; Walt, D.R. Note: Toward multiple addressable optical trapping. Rev. Sci. Instrum. 2010, 81, 026109. [CrossRef] [PubMed]

22. Schwarz, B.; Ritt, G.; Koerber, M.; Eberle, B. Laser-induced damage threshold of camera sensors and micro-optoelectromechanical systems. Opt. Eng. 2017, 56, 034108. [CrossRef] 